\title{
Linx
}

Revue des linguistes de l'université Paris X Nanterre

$54 \mid 2006$

La cause : approche pluridisciplinaire

\section{Abduction et prise en charge énonciative de la causalité}

Jean-Pierre Desclés et Agata Jackiewicz

\section{OpenEdition}

\section{Journals}

Édition électronique

URL : http://journals.openedition.org/linx/500

DOI : $10.4000 /$ linx. 500

ISSN : 2118-9692

Éditeur

Presses universitaires de Paris Nanterre

Édition imprimée

Date de publication : 1 juin 2006

Pagination : $35-47$

ISSN : 0246-8743

\section{Référence électronique}

Jean-Pierre Desclés et Agata Jackiewicz, "Abduction et prise en charge énonciative de la causalité », Linx [En ligne], 54 | 2006, mis en ligne le 01 août 2007, consulté le 19 avril 2019. URL : http:// journals.openedition.org/linx/500 ; DOI : 10.4000/linx.500 


\title{
Abduction et prise en charge énonciative de la causalité
}

\author{
Jean-Pierre Desclés, Agata Jackierwicz \\ Université de Paris-Sorbonne \\ LaLICC (UMR 8139)
}

En quelques pages, il est impensable de pouvoir traiter de toutes les expressions de la causalité que l'on pourrait trouver au travers des textes. Nous allons insister sur quelques aspects dans ce bref article, en particulier sur la relation entre l'abduction et la causalité, d'une part et d'autre part, la nécessaire prise en charge des relations causales par un énonciateur, lorsqu'on examine leur expression dans des textes.

Ces deux points semblent éloignés, mais finalement un raisonnement abductif est toujours lié à une prise en charge énonciative de celui qui oriente un raisonnement vers une cause plausible à partir d'indices dûment explicités ou explicitables, par exemple dans une justification ou une explication. Par ailleurs, l'analyse des textes montre que la causalité n'est pas toujours exprimée par des connecteurs comme parce que, puisque et car, mais également par des verbes comme provoquer, déclencher, conduire à... Cependant, il faut remarquer que les relations causales ne sont pas toujours de même nature et qu'une typologie serait souhaitable, comme nous le montrons dans la deuxième partie de l'article.

Nous commencerons par repréciser la notion d'abduction au sens de Peirce, qui conduit à formuler la plausibilité d'une hypothèse et non sa possibilité. Nous mettrons en œuvre le raisonnement abductif sous-jacent à un énoncé, qui sera repris dans la deuxième partie et opposé à des énoncés où la prise en charge énonciative est plus 
explicitement manifestée. La seconde partie insistera plus sur la construction d'une relation causale telle que l'on peut l'observer à travers sa mise en texte.

\section{Causalité et abduction}

La notion de «causalité » avec l'expression d'une justification, exprimée ou implicite, est souvent la trace d'un raisonnement abductif. Or, la notion d'« abduction » est souvent mal comprise. Le mathématicien Georges Polya, dans son ouvrage Comment poser et résoudre un problème? (1989), distingue les raisonnements démonstratifs des raisonnements heuristiques :

- Démonstratif

- Si A donc B

- Or, B est faux

- Donc, A faux

\section{Heuristique}

Si A donc B

or, B est vrai

Donc, A devient plus croyable

Le deuxième raisonnement heuristique est en fait une abduction. Georges Polya prend pour exemple le raisonnement de Christophe Colomb énoncé par :

La terre doit être proche puisqu'on voit des oiseaux.

Ce raisonnement fait appel, premièrement, à un constat d'observation : «on voit des oiseaux », puis à un savoir commun « $\mathrm{Si}$ on est près d'une terre, alors on voit des oiseaux », d'où l'hypothèse plausible : «la terre doit être proche » et, finalement, son énonciation accompagnée de sa justification.

Précisons ce que C.S. Peirce, qui a proposé le terme d'abduction, entendait par ce genre de raisonnement, qu'il opposait à l'induction et à la déduction :

L'induction généralise à partir de corrélations particulières ; son objet : formuler une loi générale.

La déduction utilise une loi générale et une prémisse; son objet : déduire une conclusion vraie à partir d'une loi vraie et d'une prémisse acceptée pour vraie.

L'abduction utilise une loi générale et un constat pour remonter à une «hypothèse causale »; son objet : remonter à une hypothèse plausible à partir d'indices identifiés.

Prenons un exemple, emprunté à Peirce. Considérons un sac qui contient des haricots et quelques haricots autour. Nous pouvons avoir les situations suivantes :

Supposons qu'un certain nombre de haricots qui ont été tirés de ce sac soient tous blancs ; donc, par généralisation inductive, « tous les haricots du sac sont blancs ».

Supposons que l'on sache que tous les haricots dans ce sac sont blancs; or, je tire un haricot du sac ; par déduction, je puis affirmer que «cet haricot est (nécessairement) blanc». 
Supposons que je sache que tous les haricots dans ce sac sont blancs ; or, voici un haricot à côté du sac, qui est percé ; par abduction, je peux en inférer que « cet haricot vient du sac » est une proposition plausible.

Nous résumons cela dans le tableau 1, ce que nous généralisons dans le tableau 2.

\begin{tabular}{|c|c|c|}
\hline $\begin{array}{l}\text { Inducti } \\
\text { Induction }\end{array}$ & $\begin{array}{l}\text { Déduction } \\
\text { Déduction } \\
\text { modus ponens }\end{array}$ & $\begin{array}{l}\text { Abduction } \\
\text { Abduction }\end{array}$ \\
\hline $\begin{array}{l}\text { foe n-ième } \\
\text { exemplaire } \\
\text { d'haricot } \\
\text { qui vient du sac } \\
\text { est encore blanc } \\
\text { pour } i=1, \ldots . .\end{array}$ & $\begin{array}{l}\quad+(\forall x) \\
\quad[\text { haricot-du-sac (x) } \\
=>\text { blanc }(x)] \\
\text { Peci est un haricot } \\
\text { tiré du sac }\end{array}$ & $\begin{array}{l}\vdash(\forall x) \\
\quad[\text { haricot-du-sac }(x) \\
=>\text { blanc }(x)] \\
\vdash \begin{array}{c}\text { Ceci est un haricot } \\
\text { blanc }\end{array}\end{array}$ \\
\hline$\vdash \forall x[\underset{=>\text { blanc }(x)}{\operatorname{haricot}(x)}]$ & † Ceci est blanc & $\begin{array}{l}\text { il est plausible que } \\
\text { cet haricot } \\
\text { vienne du sac }\end{array}$ \\
\hline
\end{tabular}

Tableau 1 : Les haricots du sac sont supposés être tirés du sac

\begin{tabular}{|c|c|c|}
\hline \multicolumn{3}{|c|}{ Induction / Déduction / Abduction } \\
\hline Induction & $\begin{array}{l}\text { Déduction } \\
\text { modus ponens }\end{array}$ & Abduction \\
\hline $\begin{array}{l}\text { F pi \& qi } \\
\text { pour } i=1, \ldots n\end{array}$ & $\begin{array}{l}-p=>q \\
F p\end{array}$ & $\begin{array}{l}-p=>q \\
F q\end{array}$ \\
\hline$F p=>q$ & - q & plausible (p) \\
\hline $\begin{array}{l}\begin{array}{l}\text { Trouver une loi par } \\
\text { généralisation de } \\
\text { corrélations particulières }\end{array} \\
\end{array}$ & 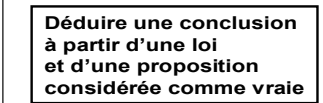 & \begin{tabular}{|l}
$\begin{array}{l}\text { Poser la plausibilité d'une } \\
\text { hypothese à partir d'une loi et } \\
\text { de constats }\end{array}$ \\
\end{tabular} \\
\hline
\end{tabular}

Tableau 2 : L'induction généralise, la déduction infère une conclusion à partir d'une prémisse supposée vraie, l'abduction infère la plausibilité d'une hypothèse à partir d'un constat.

Prenons quelques exemples très simples.

S'il pleut, alors le sol est mouillé [expression d'un savoir commun]

Or, le sol est monillé [constat]

Donc, il a plu! / Il a dû plewvoir. / Il aurait donc plu. 
Jean-Pierre Desclés, Agata Jackiewricz.

Les derniers énoncés expriment un état résultatif d'un événement passé reconstruit, par abduction jugé simplement plausible. Remarquons que la conclusion énoncée à la suite d'une abduction, peut être contestée, comme en témoigne le dialogue suivant:

- Il a dûpleuvoir!

- Pourquoi dis-tu cela?

- Eh bien, parce que le sol est monillé.

- Oui, je vois mais tu fais erreur. Si le sol est monillé, c'est parce que l'arroseuse municipale est passée dans la rue ily a quelques minutes.

- Ab bon! Je comprends.

Prenons un autre exemple :

- Tiens, le directeur est déjà arrivé!

- Pourquoi?

- Pourquoi? Mais, sa voiture est déjà au parking.

- D'accord mais ce n'est pas une raison [suffisante], d'autant, qu'bier soir, sa voiture est tombée en panne : elle n'a pas pu démarrer.

- Donc, le directeur n'est pas encore là.

- Cela m'étonnerait. Sitôt! Mais, après tout, pourquoi pas!

Le raisonnement mis en jeu dans l'énonciation de Tiens, le directeur est déjà arrivé !, se présente comme suit: appel à un savoir partagé : «lorsque le directeur est là, sa voiture est toujours au parking »; constat : «J'ai observé que la voiture du directeur est au parking », d'où (par abduction) : «Paul est arrivé » est un fait plausible, bien que, habituellement, il arrive plus tard, ce qui constitue une certaine surprise, marquée par Tiens. Ainsi, le raisonnement abductif laisse toujours place à une négociation, éventuellement à l'expression d'un doute, contrairement à l'assertion qui bloque toute négociation (Le directeur est arrivé, c'est vrai !), en mettant dans deux mondes opposés celui qui asserte et celui qui le conteste. Dans une déduction, on ne peut pas contester la conclusion dès lors que l'on a accepté comme étant vraie, ou supposée telle, la prémisse, c'est-à-dire l'hypothèse.

Reproduisons, à titre d'exemple, le raisonnement intérieur d'un juge, peu expérimenté et influençable par les seules rumeurs : « si quelqu'un est coupable alors la rumeur rapporte généralement qu'il a un comportement suspect; or, la rumeur rapporte justement que Untel a (aurait eu) un comportement suspect. Je ne puis donc pas affirmer qu'il est coupable mais la rumeur est un indice, suffisant à mes yeux, pour le considérer comme suspect ; je vais donc le mettre en examen, le procès devra alors apporter la preuve qu'il est coupable ».

Un raisonnement abductif remonte à une hypothèse ' $H$ ' à partir non pas d'un seul indice mais à partir d'un faisceau d'indices convergents :

(1) on a observé les indices $\mathrm{C}_{1}, \mathrm{C}_{2}, \ldots, \mathrm{C}_{\mathrm{n}}$;

(2) on formule une hypothèse ' $\mathrm{H}$ ' (par un acte abductif créatif) ;

(3) on se réfère à une loi générale : 'SI H ALORS on a $C_{1}$ ET $C_{2}$ ET ....ET $C_{n}$ ' 
(4) on pose, par un raisonnement abductif, la plausibilité de l'hypothèse ' $\mathrm{H}$ ', avancée comme étant une cause plausible des constats, puisque on a effectivement $\mathrm{C}_{1}$ ET $\mathrm{C}_{2}$ ET $\ldots . . . \mathrm{ET} \mathrm{C} \mathrm{C}_{\mathrm{n}}$.

Plus le faisceau d'indices ' $\mathrm{C}_{1}, \mathrm{C}_{2}, \ldots, \mathrm{C}_{\mathrm{n}}$ ' est abondant, plus la plausibilité de l'hypothèse 'H' s'en trouve renforcée. C'est pourquoi les juges, les policiers, les médecins, les archéologues, les géologues, les évolutionnistes, les historiens, les linguistes comparatistes qui reconstruisent un état de langue - non observable directement -, qui ont tous recours, dans leur pratique, à des raisonnements abductifs, chercheront à accumuler les indices pertinents, en procédant à des analyses complémentaires, en déclenchant de nouvelles observations, en recherchant de nouveaux documents, pour mieux étayer une hypothèse avancée qui deviendra ainsi, progressivement, une « cause explicative » des phénomènes constatés et décrits.

Analysons maintenant le raisonnement abductif sous-jacent à une autre énonciation :

Ce médicament a certainement agi puisque l'électrocardiogramme s'est stabilisé.

Nous devons faire appel, ici, à une analyse aspecto-temporelle, avec plusieurs référentiels temporels ${ }^{1}$, en mettant en jeu : (a) un savoir partagé général (connaissance médicale) «le cœur est en bon état si on observe que l'électrocardiogramme est stable »; (b) une relation causale exprimée dans le référentiel des situations possibles (actualisables mais non nécessairement actualisées) : «le processus impliqué par la prise du médicament recommandé CAUSE normalement un changement de l'état du cœur (qui de mauvais devient bon)». Dans le référentiel actualisé de l'énonciateur, ce dernier a une connaissance de la période temporelle déjà réalisée : (c) «l'état du cœur était mauvais » (état passé) et (d) «le malade a pris le médicament recommandé» (événement passé). Toujours dans ce référentiel énonciatif, un constat présent s'est établi : (e) «l'électrocardiogramme est maintenant stabilisé ». La conclusion s'impose alors par reconstruction abductive de l'événement plausible ( $\mathrm{f}$ ) «Le médicament a agi », cet événement est considéré alors comme étant la cause de l'état constaté «le cœur est maintenant en bon état», par le biais d'une mesure indicatrice ("l'électrocardiogramme est stable »), d'où l'énonciation, avec une marque modale : le

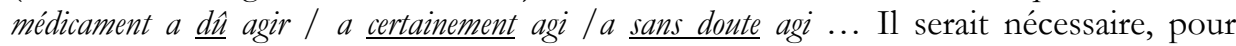
justifier complètement cette analyse, d'introduire beaucoup plus d'éléments théoriques

1 L'analyse de la catégorie aspecto-temporelle doit faire appel à plusieurs référentiels, à la fois distincts et, dans certains cas, pouvant être synchronisés. En particulier, on distingue le référentiel énonciatif construit par chaque acte d'énonciation et le référentiel externe (temps calendaire, temps cosmique...). Certains marqueurs linguistiques (un jour, ce jour lä) sont à la source d'autre référentiels (narratif ou non actualisé) dans lesquels les situations ne sont plus directement reliées à la situation énonciative de prise en charge. L'analyse de la causalité dans certains exemples, fait appel à un référentiel des possibles actualisables où sont établies des relations causales entre par exemple un processus et un changement d'état. Le raisonnement abductif va alors confronter le résultat posé de ce référentiel des possibles avec l'état effectivement constaté dans le référentiel énonciatif. C’est cette confrontation qui permettra par abduction de remonter à une cause plausible. 
aspecto-temporels ${ }^{2}$. Nous pouvons néanmoins représenter ce raisonnement qui articule des propositions relevant de deux référentiels temporels à la fois distincts et synchronisés entre eux, par le diagramme de la figure 1.

\section{Le médicament a certainement agi puisque son électrocardiogramme s'est normalisé.}

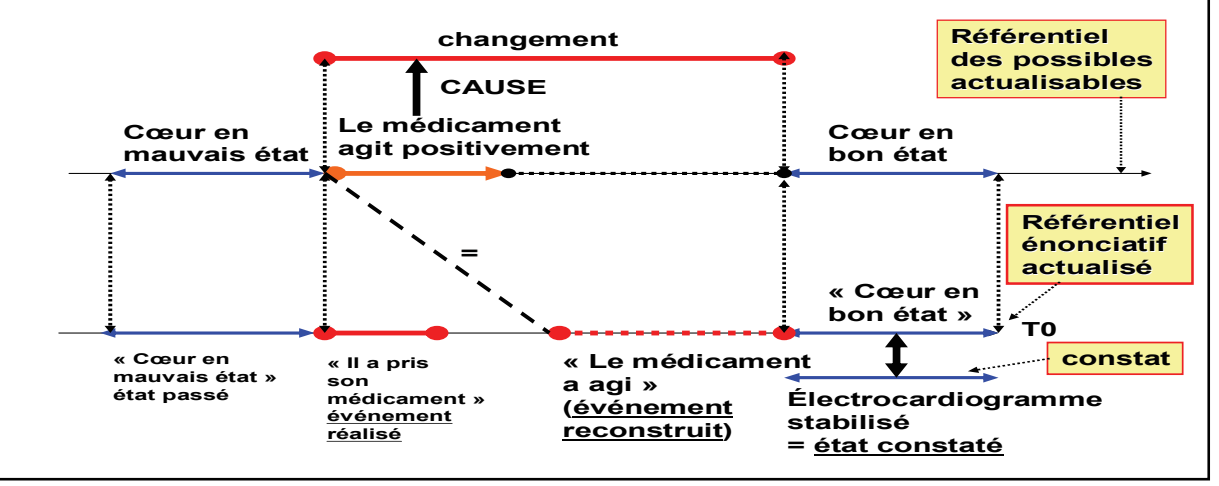

Figure 1 : Raisonnement abductif en médecine concluant à une cause plausible

\section{Causalité et prise en charge énonciative}

Comment les moyens d'expression langagiers rendent-ils compte des représentations des relations causales qu'un locuteur humain se fait ou construit et choisit de transmettre? Nous défendons l'hypothèse (Jackiewicz 1998, 1999, 2004) que la causalité encodée dans la langue peut être vue comme une relation complexe intégrant d'une part, une relation efficiente entre situations et d'autre part, une relation subjective entre cette relation efficiente et le locuteur qui la prend en charge. Les différentes modalités de prise en charge énonciative présentes dans les énoncés causaux sont à divers degrés liées, tant conceptuellement que sur le plan de l'expression avec la notion de causalité.

\subsection{Carte cognitive de la causalité}

Les études linguistiques portant sur la causalité sont menées traditionnellement à l'intérieur de deux champs distincts. Le premier s'intéresse à l'argumentation et donc à l'organisation raisonnée du discours. Cette organisation est marquée entre autres par certains connecteurs considérés comme causaux, tels que parce que, puisque et car. Le deuxième champ d'investigation est celui de la sémantique lexicale, dont l'objet est la description de verbes qualifiés d'efficients ou de causatifs, selon le mode de participation du sujet à l'action, donc exprimant une certaine agentivité.

2 Nous renvoyons aux articles relatifs à la catégorie de l'aspectualité de J.P Desclés et de Z. Guentcheva. 
Ces deux approches de la causalité, fort différentes entre elles, reflètent assez nettement la distinction que la langue opère entre la causalité que l'on établit et la causalité que l'on exploite (figure2). En effet, la langue semble réserver les moyens grammaticaux ou grammaticalisés à l'expression des enchaînements discursifs fondés sur des liens causaux bien établis, notamment sur des lois causales communément partagées, souvent implicites (a). Elle fait appel à des moyens lexicaux (essentiellement verbaux), plus riches en possibilités d'expression, notamment pour exprimer différentes manières d'agir, quand il s'agit de construire une telle relation ou d'en parler (b).

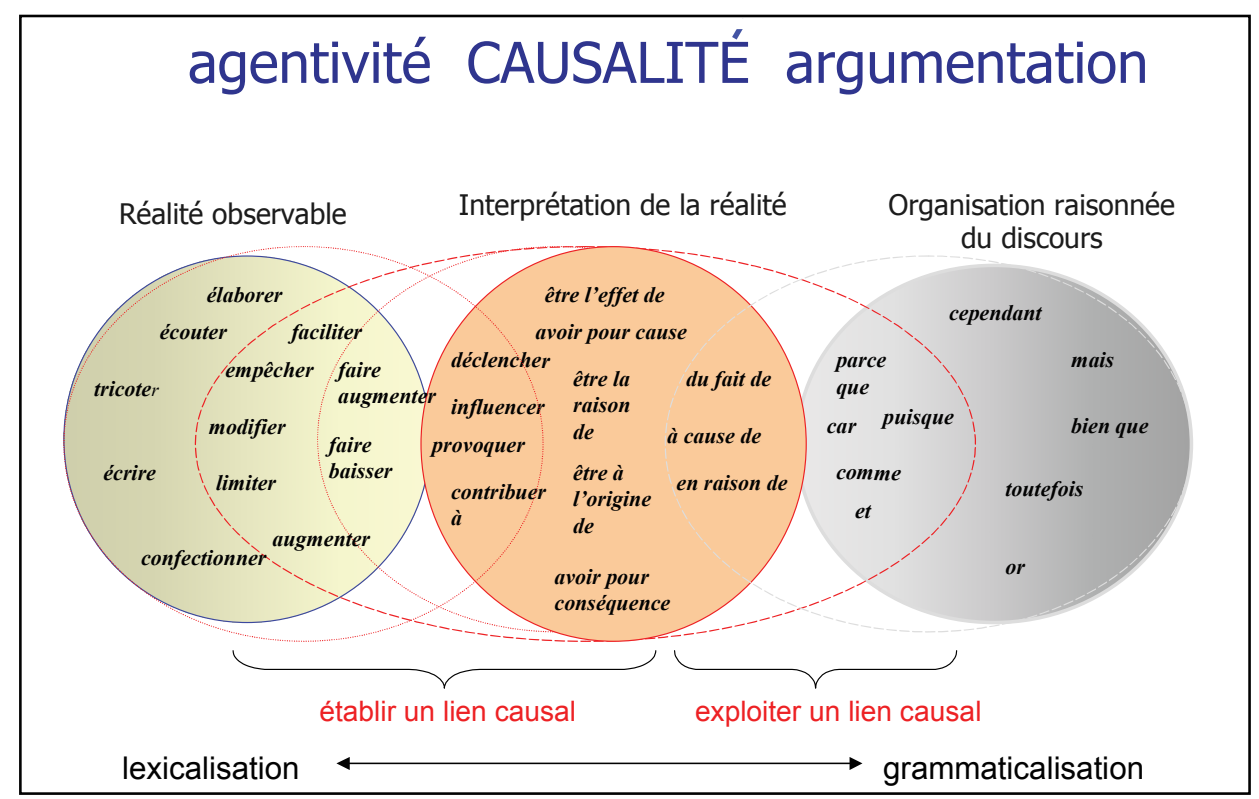

Figure 2 : Articulation causalité - agentivité - argumentation

(a) Ce médicament a certainement agi puisque l'électrocardiogramme s'est stabilisé.

(b) On sait que la cataracte - première cause mondiale directe de cécité absolue - est provoquée par une lésion de l'œil, qui peut être le fait, entre autres facteurs, d'une pollution aérienne lourde.

Considérons maintenant la partie gauche de la carte, pour nous intéresser aux moyens d'expression lexicalisés, et notamment aux verbes qui sont situés à l'intersection des domaines de l'agentivité et de la causalité. Ce très vaste ensemble de verbes efficients permet d'exprimer tant une action intentionnelle effectuée avec contrôle et téléonomie qu'une action privée de toutes ces propriétés. Avant de considérer les exemples illustratifs de ce phénomène de polysémie, précisons les critères définitoires des notions d'agentivité et de causalité.

Les énoncés agentifs expriment une situation unique avec deux participants (l'agent et le patient) et un état résultant. L'agent qui effectue une action affectant le patient n'est pas en relation causale avec l'état résultant du patient. Les capacités d'effectuation directe, de contrôle et de téléonomie qu'il met en œuvre dans l'action, 
Jean-Pierre Desclés, Agata Jackiewricz.

sont autant de propriétés qui rendent son acte difficilement séparable de l'effet produit. Un exemple d'énoncé agentif prototypique serait Marie écrit un poème. La causalité, quant à elle, sera vue comme une relation entre deux situations que l'on peut appréhender, conceptualiser et verbaliser d'une manière indépendante. Une situation par excellence est un état, un processus ou un événement (une lésion de l'cil dans b), mais aussi toute entité susceptible d'une interprétation processuelle ou événementielle (une pollution, une mer... qui ravage les côtes).Regardons comment les verbes efficients interagissent avec leurs arguments et avec d'autres éléments, notamment modaux, présents dans leurs contextes. Quand les arguments correspondent à des processus, la lecture causale s'impose $(1,2)$.

(1) Sécheresse et incurie politique provoquent une famine en Afrique.

(2) Les délocalisations d'activités industrielles vers Pékin et Shanghaï augmentent les inquiétudes des Européens.

Dans le cas où le premier argument, exprimant l'entité qui agit, est une entité intentionnelle a priori, deux lectures sont possibles selon le contexte. Une lecture préférentiellement agentive pour l'énoncé 3, où l'on note l'influence d'une modalité d'action (inchoactive) commencer à. Une lecture causale, dans (4), où la présence de l'adverbe modal incontestablement focalise l'information sur l'existence possible (incontestable ?) d'un lien entre l'attitude de certains enseignants et la dépression scolaire.

(3) Les conseillers économiques de Clinton commencèrent dès février 1994 à provoquer la baisse du dollar.

(4) L'anxiété de l'élève est constituée de trois éléments: la tendance anxieuse, l'anxiété de sa famille, l'anxiété (éventuelle et diversement exprimée) des maîtres. Incontestablement, quelques enseignants - de moins en moins nombreux - provoquent une sorte de 'dépression' scolaire.

Quand l'argument qui exprime l'entité qui agit est tout simplement un objet ou une entité, nous avons à choisir entre une lecture instrumentale, qui semble s'imposer pour l'énoncé 5 , et une lecture causale, dans l'exemple 6 .

(5) La science évolue sans cesse et les laboratoires travaillent sans relâche sur de nouveaux moyens capables de modifier les comportements et d'augmenter les performances. (...) Si les produits dopants augmentent la masse musculaire...

(6) Pensez-vous que les produits financiers de plus en plus sophistiqués angmentent la volatilité des marchés ou, au contraire, qu'ils ont permis aux institutions financières d'absorber plus facilement le kerach?

En effet, les produits dopants, comme le précise le contexte qui précède la proposition, sont fabriqués pour augmenter les performances des sportifs. Ils constituent un instrument employé dans une visée précise. L'action des produits financiers fait, quant à elle, l'objet d'un questionnement. L'augmentation de la volatilité des marchés, comme effet, est un effet possible qui est discuté et pesé dans la suite du texte. 
$\mathrm{Au}$ sein de la relation prédicative, on observe donc une interaction (un ajustement mutuel) entre les significations respectives des différents lexèmes qui jouent le rôle de prédicat, d'arguments et de modalités. Parmi les éléments qui interviennent dans la caractérisation distinctive de la notion de causalité par rapport à celle d'agentivité, les modalités méritent à nos yeux une attention particulière. Il s'agit d'une part des modalités d'action qui spécifient le degré d'implication de l'agent humain dans l'action qu'il effectue (elle peuvent toucher à l'intentionnalité, au contrôle, à la téléonomie, à la manière de faire, à l'appréciation normative). D'autre part, il y a des modalités de prise en charge qui focalisent l'attention sur la plausibilité et sur l'intelligibilité du lien établi entre deux situations distinctes.

\subsection{La causalité prise en charge}

Plusieurs linguistes ont signalé, montré ou exploité le lien qui existe dans la langue entre la catégorie des modalités et celle de la causalité. Citons le constat fait à ce sujet par Ferdinand Brunot.

Il est peu de rapports qu'on ait plus souvent besoin de mettre en relief. Plus le rapport de causalité entre deux faits est douteux, plus on peut avoir besoin d'y insister (Brunot 1922, p. 823).

Le rapport de cause peut être lui-même dans toutes sortes de modalités; on peut le présenter comme certain, possible, irréel. Pour assurer, on dira: c'est sûrement, certainement... parce que. Pour présenter avec réserve au contraire, on se servira des moyens ordinaires : peut-être, sans doute: s'il est si timide, c'est peut-être manque d'babitude (Brunot 1922, p.827).

Leonard Talmy (1988), quant à lui, regroupe les catégories de causation et de modalité dans une catégorie plus générale à ses yeux que constitue la dynamique des forces.

Notons également que la terminologie causale est, elle-même, riche d'expressions qui renvoient, soit à une construction intellectuelle, soit à un jugement subjectif : imputation causale, impression de causalité, suspicion de causalité, interprétation causale, assignation causale, assertion du lien causal, attribution causale, jugement de causalité, degré de certitude du lien causal, détection de causalité, établissement du lien causal....

La prise en charge énonciative est une notion linguistique qui représente la dimension modale (modus) emboitant la dimension prédicative (dictum) de l'énoncé. C'est une opération complexe, qui comme l'ont montré (Desclés et Guentchéva 2000), peut se décomposer en plusieurs opérations élémentaires, paraphrasables comme suit :

- Je dis que ce qui est dit est vrai (assertion);

- Je dis que ce qui est dit est vrai selon une certaine modalité (possible...);

- Je dis que ce qui est dit est vrai dans un contexte déterminé ;

- Je dis que ce qui est dit est dit par un autre, déterminé ou indéterminé (médiation);

- Je dis que ce qui est dit est inféré à partir d'indices ;

- Je dis que ce qui est dit est attesté par un témoin (vu ou entendu). 
En français, quand le dictum exprime une relation causale, ce sont les quatre premières opérations énonciatives qui l'on atteste en priorité. Chacune d'entre elles colore différemment l'information causale exprimée et rend compte d'une vision particulière qu'un énonciateur se fait de cette relation et choisit de transmettre.

L'énonciateur produit souvent des assertions de relations causales qui sont pleinement assumées par lui. Cette prise en charge peut prendre plusieurs nuances, selon la manière dont l'énonciateur choisit de légitimer le savoir causal qu'il exprime. L'assertion peut être fondée sur un constat empirique direct, lié à la perception. Elle peut puiser sa légitimité dans le fait d'être assumée collectivement par une communauté déterminée. Elle peut aussi s'appuyer sur le fait que l'énonciateur tient le lien causal pour explicable ou intelligible. L'adhésion au contenu prédicatif atteint son degré maximal quand l'énonciateur déclare être lui-même à l'origine de la relation prédicative exprimée.

(7) De telles difficultés m'ont alors semblé révélatrices d'une faiblesse de notre démocratie dont j'attribue la cause à une politique constante d'éducation nationale qui mériterait d'être corrigée.

Le savoir causal est souvent asserté comme dépendant d'un point de vue ou d'un contexte particulier, qui déterminent ainsi son cadre de validité. Ce type d'assertion contextualisée est toujours assumé par l'énonciateur. Celui-ci assume directement non pas la relation causale, mais le fait de la rendre dépendante d'un contexte. Plusieurs cas de figure, relativement proches les uns des autres, peuvent se présenter selon les circonstances dans lesquelles le contenu prédiqué peut être considéré comme valide. Une connaissance causale peut être vraie uniquement à l'intérieur d'un cadre spatial ou temporel, au sein d'une discipline ou une théorie particulière, ou encore peut n'être tenue pour fondée que par certaines personnes ou par certains groupes de personnes (critère énonciatif).

Le contexte de validité restreint permet de comprendre pourquoi plusieurs explications causales différentes (voire concurrentes) peuvent être proposées en même temps, chacune relativement à son contexte (relativité explicative). Les rédacteurs ont en général à cœur que leurs lecteurs reconnaissent correctement les limitations de validité du savoir transmis.

(8) Dans la théorie standard, dite "néoclassique ", le libre fonctionnement du marché conduit l'économie dans son ensemble à un état optimal.

La prise en charge médiatisée introduit un tiers plus ou moins déterminé. L'énonciateur prend de cette façon de la distance par rapport à ce qu'il énonce. $\mathrm{Ne}$ pouvant être légitimées par une origine reconnue, les relations causales ainsi médiatisées sont généralement incertaines et restent à vérifier. Pour exprimer un désengagement, l'énonciateur peut recourir au conditionnel, ainsi qu'à des expressions telles que d'après, selon les rumeurs; il paraît que, il paraîtrait que; paraît-il (en incise) ; dit-on (en incise); le bruit court que.

(9) Une enquête de l'Assistance publique de Paris révélait en mars 1993 que près de $10 \%$ de malades étaient victimes d'une infection contractée durant leur séjour. D'après diverses sources, cette calamité serait responsable de six mille à dix-buit mille morts par an auxquels on peut 
ajouter les accidents thérapentiques qui feraient quelque dix mille victimes selon l'Association des victimes d'actes médicaux.

Le contenu d'une proposition causale peut également se trouver modifié par une idée de nécessité, d'impossibilité, de possibilité ou de contingence. On peut noter par exemple que les modalités du possible et du nécessaire renforcent la réalité du lien causal en le présentant comme inhérent aux choses. Leur présence dans un énoncé causal traduit généralement une efficience causale latente, plutôt réalisable que réalisée.

(10) La tyramine qui est un facteur biochimique migrainogène cité plus haut est susceptible de provoquer des crises de migraine authentique et il faut connaître les aliments riches en tyramine.

Quand un énoncé causal est modifié par une modalité épistémique, il rend compte d'un état de connaissance ou d'un degré de certitude de l'énonciateur par rapport à la réalité du lien causal prédiqué « je dis que ce qui est dit est certain ou probable ou improbable...». L'énonciation causale modalisée par des modalités de fréquence ou d'habitude suppose un savoir sur des événements déjà accomplis et marque la possibilité d'extrapolation sur du encore possible.

La présence significative des éléments modaux dans l'expression de la causalité, nous amène à considérer la causalité langagière comme une relation complexe qui intégrerait deux relations constitutives, d'une part une relation efficiente entre deux situations (R1), et d'autre part, une relation subjective (R2) entre cette relation efficiente et celui qui la prend en charge $(\mathrm{H})$.

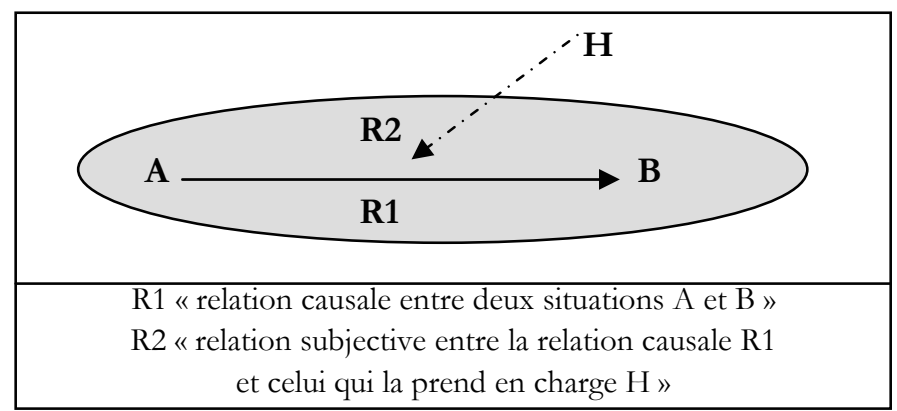

Figure 3 : Double dimension de la causalité dans la langue

De nombreux marqueurs témoignent de différentes manières de cette double dimension de la causalité dans la langue. Certains encodent directement la relation complexe, où R1 et R2 sont intriquées (11a). Dans (11b) et (11c), la co-présence de R1 et de $\mathrm{R} 2$ est plus transparente, notamment grâce à la co-présence du prédicat verbal qui exprime le lien causal et d'un marqueur qui exprime une certaine modalité portant sur le contenu prédicatif.

(11a) Arrbénius lie définitivement la dérive de l'effet de serre et l'utilisation des combustibles fossiles.

(11b) L'ingestion de vin aurait un effet protecteur sur l'organisme.

(11c) Selon Freud, l'oubli est le fruit d'un refoulement. 
Jean-Pierre Desclés, Agata Jackiewricz.

La relation subjective et la relation efficiente sont étroitement liées entre elles. Tout d'abord, la relation subjective peut modifier sensiblement le contenu de la relation efficiente, ensuite ces deux relations sont souvent indissociables sur le plan de l'expression, enfin, la relation subjective guide l'interprétation de la relation efficiente quand cette dernière est exprimée par des marques polysémiques.

La dimension modale, si présente dans l'expression de la causalité, rend compte des représentations de cette notion que le locuteur humain se fait ou construit et choisit de transmettre. La causalité nait de l'interprétation du monde. L'énonciateur ne se contente pas de « lire » le monde qui l'entoure, mais cherche activement à construire des relations à la fois signifiantes et opératoires. Et cette causalité, laquelle se construit le plus souvent collectivement et progressivement, apparait dans la langue comme une notion dialogique. 


\section{RÉFÉRENCES BIBLIOGRAPHIQUES}

BRunOt F., 1922, La pensée et la langue, Paris, Masson.

DesClÉs J.-P., 2000, « Abduction and Non-observability - Some Examples from Language and Cognitive Sciences », in Avandro Agazzi and Massimo Pauri, The Reality of Unobservable, Observability, Unobservability and their Impact on the Issue of Scientific Realism, Kluwer Academic Publishers : 87-112.

Desclés J.-P. \& GuentChÉva Z., 2000, «Enonciateur, locuteur, médiateur », in A. Becquelin et $\mathrm{Ph}$. Erikson ed., Les rituels du dialogue, l'Harmattan,.

JACKIEWICZ A., 1998, L'expression de la causalité dans les textes. Contribution au filtrage sémantique par une méthode informatique d'exploration contextuelle. Thèse de doctorat, Université de Paris Sorbonne,

JACKIEWICZ A., 1999, "Causalité et prise en charge énonciative», Etudes Cognitives, n $3: 249$ 269, Académie polonaise des Sciences, Varsovie, Pologne,.

JACKIEWICZ A., 2004, «La causalité dans la langue : une question de point de vue », Intellectica, $\mathrm{n}^{\circ} 38$.

SANDers Peirce C., Selected Papers, Harvard.

POLYA G., 1989, Comment poser et résoudre un problème ?, Editions Jacques Gabay,.

TAlmy L., 1988, "Force Dynamics in Language and Cognition », Cognitive Science, Vol. 12 : 49100. 
